\title{
Stereological Study of Collagen and Elastic System in the Detrusor Muscle of Bladders from Controls and Patients with Infravesical Obstruction
}

\author{
Mauricio Rubinstein, Francisco J. Sampaio, Waldemar S. Costa \\ Urogenital Research Unit, State University of Rio de Janeiro, Rio de Janeiro, Brazil
}

\begin{abstract}
Objective: Compare detrusor muscle of normal and patients with infravesical obstruction, quantifying the collagen and elastic system fibers.

Materials and Methods: We studied samples taken from bladders of 10 patients whose ages ranged from 45 to 75 years $($ mean $=60$ years $)$, who underwent transvesical prostatectomy for treatment of BPH. Control material was composed of 10 vesical specimens, removed during autopsies performed in cadavers of accident victims, with ages between 18 and 35 years (mean $=26$ years).

Results: The results of collagen and elastic fibers quantification (volumetric density) demonstrated the following results in percentage (mean +/- standard deviation): collagen in BPH patients $=4.89+/-2.64$ and $2.32+/-1.25$ in controls $(\mathrm{p}<$ $0.0001)$, elastin in BPH patients $=10.63 \%+/-2.00$ and $8.94 \%+/-1.19$ in controls $(\mathrm{p}<0.0001)$.

Conclusion: We found that the components of connective tissue, collagen and elastic system fibers are increased in the detrusor muscle of patients with infravesical obstruction, when compared to controls.
\end{abstract}

Key words: bladder, prostatic hyperplasia, bladder outlet obstruction, collagen, elastin

Int Braz J Urol. 2007; 33: 33-41

\section{INTRODUCTION}

The benign prostatic hyperplasia $(\mathrm{BPH})$ is a pathologic process that contributes but is not the only cause of lower urinary tract symptoms in men. It is recognized today that part of the symptoms in men with BPH may be the result of a detrusor muscle dysfunction related to age (1).

In patients with bladder outlet obstruction (BOO), in addition to hypertrophic changes in smooth musculature, other changes related to the collagen and to the elastic system fibers were already evidenced. However, most of theses studies were conducted on animals (2-4).
Kondo \& Susset (2) did not verify changes in collagen in bladders of dogs subjected to infravesical obstruction. On the other hand, an increase in the amount of collagen was described by Dixon et al. (4) in bladders of swine subjected to partial urethral obstruction.

Uvelius \& Mattiasson (3) described in rats, the decrease in the amount of collagen in detrusor muscle hypertrophy caused by infravesical obstruction.

Nielsen et al (5) subjected 9 pigs to chronic partial infravesical obstruction through an urethral ring. The results showed an 8 -fold increase in total collagen and in type I and III collagen. 
Collagen and elastic fibers are the main components of extracellular matrix; they are present in the bladder wall and are closely related to vesical compliance (6). The increase of collagen and other components of extracellular matrix occur as a tissue response to injury and can lead to fibrosis of the organ. Previous studies have demonstrated that collagen synthesis is increased in pulmonary fibrosis (7), in glomerulosclerosis (8) and in other pathologies.

Congenital or acquired infravesical obstruction can result in a fibrotic vesical wall, characterized by trabeculation, increase of connective tissue, low volumetric capacity under high pressures and, in some cases, the occurrence of non-inhibited contraction of detrusor muscle. The vesical wall can accumulate connective tissue and evolve to a fibrotic process in consequence of a neurologic (myelomeningocele, spinal cord lesion) or obstructive $(\mathrm{BPH})$ process. Such pathological conditions can result in low capacity, high pressure and low vesical compliance (9).

This study aims to compare bladders of normal patients and those with infravesical obstruction, quantifying by stereological and computer-assisted methods the collagen and elastic system fibers of detrusor muscle.

\section{MATERIALS AND METHODS}

We studied samples with approximately $2 \mathrm{x}$ $2 \mathrm{~cm}$, including all the layers of vesical dome, taken from bladders of 10 patients whose ages ranged from 45 to 75 years (mean $=60$ years), who underwent transvesical prostatectomy for treatment of $\mathrm{BPH}$. The prostatic mass removed weighted $95 \pm 10$ grams in average. Previously to surgery, all patients were subjected to an urodynamic study in order to confirm and characterize the presence of BOO. A detrusor pressure above $100 \mathrm{~cm}$ of $\mathrm{H}_{2} \mathrm{O}$ and an urinary flow lower than $10 \mathrm{ml} / \mathrm{s}$ were considered as urodynamic evidence of BOO. The methodology and definitions used in the work complied with the International Continence Society standards (10). All patients who presented vesical instability on the urodynamic examination were excluded from the study. In all cases an informed consent was obtained with the patient for removal of vesical samples.

Control material was composed of 10 vesical specimens, removed during autopsies performed in cadavers of accident victims, with ages between 18 and 35 years (mean $=26$ years). The autopsy was performed up to 6 hours after death, and it was verified that there was no compromise of urogenital system organs. The removed fragments included all layers of vesical dome and measured about $2 \times 2 \mathrm{~cm}$. The study protocol was approved by the Committee on Human Research of the State University of Rio de Janeiro.

Immediately after the removal, the material was fixed in buffered formalin $10 \%(\mathrm{pH}=7.2)$ during 48 to 72 hours, and subsequently underwent a routine histological processing for inclusion in paraffin. The resultant blocks were then subjected to serial sections of 5- $\mu \mathrm{m}$ thickness, obtaining 10 sections from each bladder fragment.

Sections were stained by Picro-Sirius Red technique for evidencing collagen and by Weigert's Fuchsin-Resorcin for characterizing the elastic system fibers.

Image acquisition and analysis - Five sections were randomly analyzed, from each bladder, and in each section, 5 fields were analyzed, totaling 15 fields per bladder.

Quantification of the elastic system was made with sections stained by Weigert's Fuchsin-Resorcin. The analyzed fields were digitized to a final magnification of $x 400$ using a video camera coupled to a light microscope. The alleatory histological areas were quantified using a M-42 test-grid system on the digitized fields on the screen of a color monitor. The stereological method has been described in detail elsewhere $(11,12)$.

For quantification of collagen fibers the sections stained by Picro-Sirius Red technique were observed under polarized light (13). The analyzed images were obtained with a magnification of $x 400$ using a video camera coupled to a light microscope.

The stereological parameter employed for estimating the contents of extracellular matrix (collagen and elastic system fibers) was volumetric density $(\mathrm{Vv})$. Mean values of $\mathrm{Vv}$ were calculated for 
both groups in relation to elastic fibers and collagen and subsequently compared.

Data were analyzed on Graphpad InStat software (Graphpad). Mann-Whitney test was used for analysis of results, considering a value of $p<0.05$ as significant.

\section{RESULTS}

The observation of histological sections showed an increase of collagen and elastic system fibers in the detrusor muscle of patients with infravesical obstruction, compared to the controls (Figure-1).

The results of quantification of collagen and elastic system fibers are presented on Table-1.

\section{DISCUSSION}

The morphology of normal human bladder has been studied and compared to the obstructed bladder and to the elderly bladder through optic and
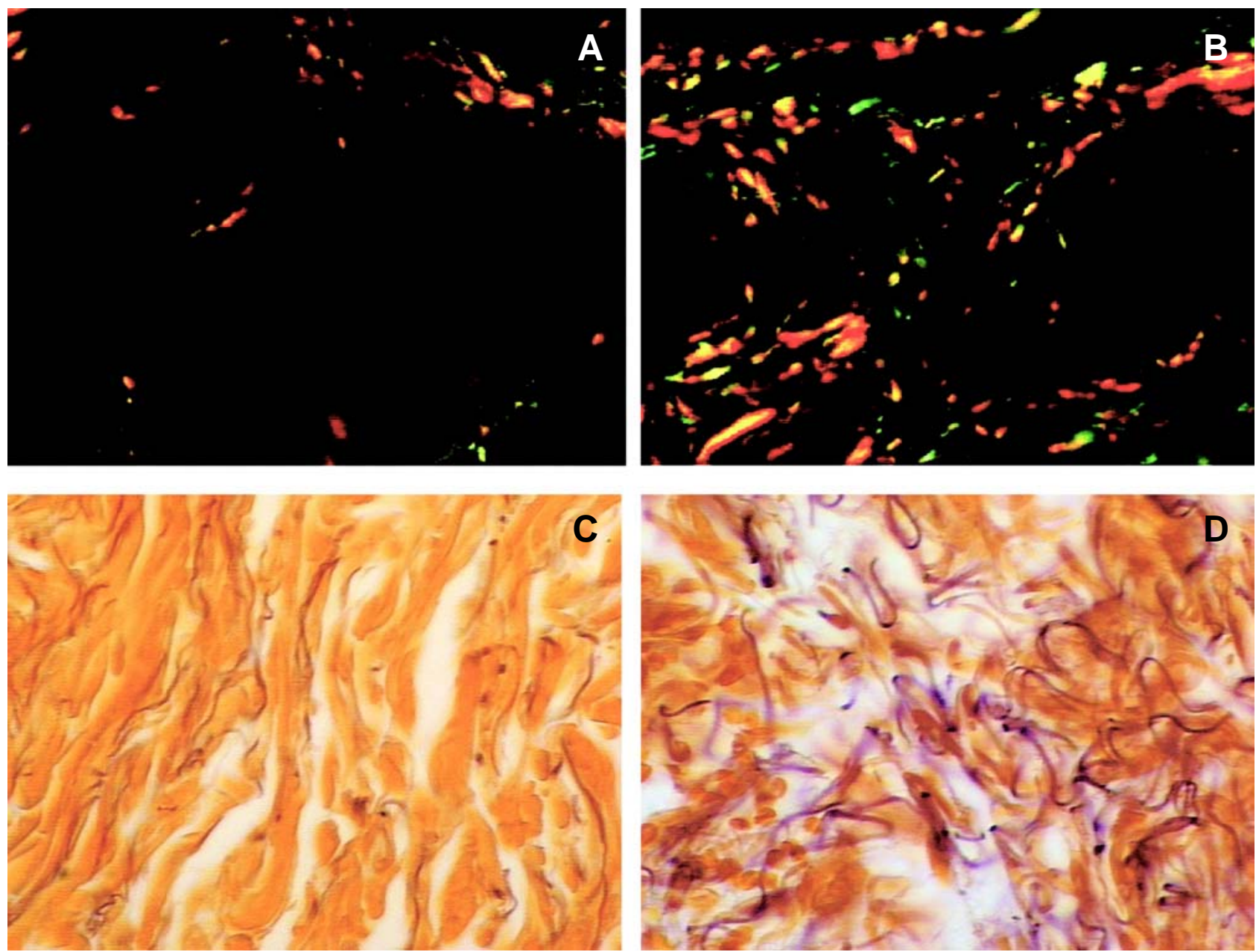

Figure 1 - Picro-Sirius-Red staining under polarization microscopy of the bladder wall demonstrating the amount of collagen in controls $(A)$ and patients with bladder outlet obstruction (B), X400. Weigert's Resorcin-Fucsin staining of the bladder demonstrating the amount of elastic system fibers in controls $(C)$ and patients with bladder outlet obstruction $(D), X 400$. 
Table 1 - The volumetric density in percentage of collagen and elastic system fibers in the bladder of controls $(n=10)$ and benign prostate hyperplasia $(B P H)$ samples $(n=10)$.

\begin{tabular}{lcccc}
\hline & \multicolumn{2}{c}{ Collagen } & \multicolumn{2}{c}{ Elastic System Fibers } \\
& Controls & BPH & Controls & BPH \\
\hline Mean & 2.32 & 4.89 & 8.94 & 10.63 \\
Standard deviation & 1.25 & 2.64 & 1.19 & 2.00 \\
Standard error & 0.39 & 0.83 & 0.37 & 0.63 \\
Minimum & 0.73 & 1.96 & 7.14 & 8.25 \\
Maximum & 4.31 & 8.27 & 10.63 & 14.12 \\
P value & \multicolumn{2}{c}{$<0.0001$} & \multicolumn{2}{c}{$<0.0001$} \\
\hline
\end{tabular}

electronic microscopy $(6,14)$. In the bladder of patients without urologic disease, muscle fibers are grouped with few connective tissue between their bundles. In the obstructed bladder, however, many muscle fibers are surrounded by connective tissue bundles, and they also change their function, becoming involved in collagen synthesis (15).

Kim et al (16) analyzed in rats the effects of partial obstruction of bladder over type I and III collagen, and the relationship between detrusor contractility and types of collagen. The results showed an increase in total collagen following infravesical obstruction and a decrease following the obstruction's relief. This work suggests that a change in localization and quantity of collagen leads to vesical alterations, which can impair detrusor contractility. In our work, conducted in humans and employing a different methodology, confirms the findings by Kim et al. (16), demonstrating a significant increase not only in collagen but also of elastic system fibers in patients with vesical obstruction.

Cortivo et al. (6) evaluated the connective tissue of bladders of normal patients and patients with infravesical obstruction. Three distinct groups were assessed: 1) 3-month children with infravesical obstruction, 2) children between 4 to 8 years with partial infravesical obstruction and 3) adults with chronic infravesical obstruction. An increase of elastic tissue occurred in newborns and in adults with obstruction. Collagen did not present any changes between the groups under study. In this work, according to the authors, collagen and elastic fibers were not quantified. In our current study an important increase of elastic tissue was observed, corroborating, partially, the results from Cortivo et al. (6), however, differently from their work (6), our results demonstrated a significant increase of collagen in the detrusor muscle of patients with obstructive BPH.

Gosling \& Dixon (17) compared microscopic findings from biopsies of the detrusor muscle with the grade of vesical trabeculation assessed by cystoscopy. The author observed an important infiltration of connective tissue elements in the detrusor muscle of patients with severely trabeculated bladders. Data from our current work, also show that collagen surrounds and infiltrates the muscle bundles, suggesting that fibrous elements can lead to a reduction of compliance in bladders of patients with infravesical obstruction.

Gilpin et al. (9) while studying the morphology and morphometry of detrusor muscle fibers from patients with obstruction, described structural changes of the vesical wall, represented by collagen deposit in muscular interstice, and by the increase in volume of muscle fibers, what is in accordance to our results.

Freedman et al. (18) demonstrated that the relationship between smooth muscle versus connective tissue remained the same in control (normal) and obstructed fetal bladders. These findings show that the amount of collagen associated with infravesical obstruction is also a controversial subject in human bladders.

Lepor et al. (19), with the purpose of characterizing the effects of aging and of obstructive 
$\mathrm{BPH}$ over the vesical wall, conducted a retrospective study in 86 vesical samples, obtained from medical files, and removed during autopsies. The study was divided into 4 groups: 1) men aged between 35 and 45 years; 2) men aged between 65 and 75 years; 3 ) women aged between 35 and 45 years; and 4) women aged between 65 and 75 years. The groups were divided in order to select men with $\mathrm{BPH}$ and without $\mathrm{BPH}$. There was a significant increase of density in the smooth muscle and connective tissue area in group 1 vs. group $2(2.90+/-0.22$ vs. $2.33+/-0.16)$ and in group 3 vs. group $4(2.85+/-0.13$ vs. $2.03+/-0.20)$. The increase in the connective tissue ratio was agedependent in both female and male groups. Based on these results, the authors suggest that aging, and not $\mathrm{BPH}$, is related to an increase of fibrosis in the detrusor muscle. Nevertheless, one must take into account that the examined material in this study came from patients who did not undergo urodynamic tests, that would confirm or not the presence of infravesical obstruction. In our study, the previous conduction of an urodynamic assessment confirmed the existence of an obstructive process in all the patients under study. Our results showed that there was a significant increase of collagen and elastic fibers when the groups of men with BPH and without BPH were compared.

The study by Holm et al. (20) questions if the fibrosis of the detrusor muscle is a consequence of infravesical obstruction, of the patient's age, or both factors. Morphological studies of vesical wall were performed through biopsies from young patients without disease, patients with infravesical obstruction, and elderly without obstruction. It was observed fibrosis inside and around the muscle fascicles, in the same proportion in obstructed patients and in elderly. The results from this work pose the question whether the fibrotic changes of detrusor muscle occur due to obstruction itself, to aging, or both.

Gosling (15) demonstrated that in the elderly, the bladder differs from the obstructed bladder regarding the collagen deposit. In the elderly bladder there is no evidence of collagen synthesis by smooth muscle fibers. On the other hand, in the bladder that is obstructed by BPH there is an accumulation of connective tissue in the detrusor muscle, where several smooth muscle fibers change their function, and become more involved with the collagen synthesis. Thus, the author concluded that it is unlikely that the changes occurring in the bladder of patients with obstruction merely reflect alteration due to aging. The results of the current work ratify these data (15), objectively demonstrating that, in the bladder obstructed by BPH, there is an accumulation of connective tissue in the detrusor muscle.

Deveaud et al. (21) analyzed 45 children who underwent surgical treatment for non-compliant and fibrotic bladders due to a number of causes (myelomeningocele, posterior urethral valve, vesicoureteral reflux). Vesical fragments were analyzed through immunohistochemical for localizing collagen. A major infiltration of connective tissue was evidenced in detrusor's smooth muscle bundles. The results showed an increase in total collagen, through dosing of total hydroxyproline content, and a significant increase in type I and III collagen. The work suggests that the collagen deposit in the detrusor muscle is a marked histological characteristic of noncompliant bladders, regardless of their etiology. Our results show a concordance with this work (21) even though the methodology and groups under study were different. In our study, even though a separate quantification of type I and type III collagen was not performed, the greenish color present in the sections observed under polarization microscopy is highly expressive and suggests the presence of a significant amount of type III collagen.

It is important to highlight that the majority of previous studies, in humans, were conducted without confirmation of an obstructive condition by urodynamic tests.

Inui et al. (22) observed changes in connective tissue of the detrusor muscle of patients with obstructive BPH. Thirteen normal men with ages ranging from 25 to 90 years $(68.5+/-18)$ and 26 cases of BPH patients with ages between 60 and 94 years (71.6 +/- 9.2) were studied. BPH diagnosis was made based on the prostate transrectal ultrasonography. The mean values for connective tissue and smooth muscle between control and BPH cases were subsequently compared through computer assisted color image analysis. The authors found that abnormal increase of connective tissue in addition to smooth muscle 
hypertrophy and/or hyperplasia could contribute to advanced bladder hypertrophy caused by infravesical obstruction. Our findings confirm the increase of connective tissue in patients with $\mathrm{BPH}$ and demonstrate a significant difference in the percentage of collagen and elastic system fibers between the control and BPH groups.

Recently, Collado et al. (23) studied by computer assisted morphometry the detrusor muscle cell diameter and the connective tissue-to-smooth muscle ratio in patients with bladder outlet obstruction, acute urinary retention and a nonobstructed control group. The author found morphometric differences in detrusor muscle cell diameter and the connective tissue-to-smooth muscle ratio between controls and patients with obstruction. Also, it was found an increase in detrusor muscle cell diameter and fibrosis in bladder outlet obstruction, which are in agreement with our findings concerning extracellular matrix components.

The understanding of changes consequent to a response to vesical injury, may allow the development of strategies to prevent the pathologic remodeling of extracellular matrix observed in diseased bladders. Obstructive processes, diseases related to age, or even aging itself, can influence vesical function. The relationship between obstruction and aging has not been completely clarified. The lower urinary tract symptoms are not specific, and alone they do not enable the diagnosis of obstruction. The symptomatology in certain conditions, such as vesical instability and detrusor hypocontractility without obstruction, mimic those of obstructive $\mathrm{BPH}$, but there is no proof that such conditions result solely from aging.

Even if we know that some changes occurring in the bladder that is subjected to infravesical obstruction are possibly related to aging processes, a comparison with bladders of normal young individuals, such as the one we performed on this work, can be useful for establishing a pattern of the different vesical components, which will be modified either by aging or by pathologic processes.

In conclusion, we found that the components of connective tissue, collagen and elastic system fibers are increased in the detrusor muscle of patients with infravesical obstruction.

\section{ACKNOWLEDGMENTS}

Supported by grants from the National Council of Scientific and Technological Development $(\mathrm{CNPq})$, and Foundation for Research Support of Rio de Janeiro (FAPERJ).

\section{CONFLICT OF INTEREST}

None declared.

\section{REFERENCES}

1. Elbadawi A: Functional anatomy of the organs of micturition. Urol Clin North Am 1996; 23: 177-210.

2. Kondo A, Susset JG: Collagen content in detrusor muscle and ratio of bladder weight to body weight. Acta Urol Jap 1973; 19: 683-686.

3. Uvelius B, Mattiasson A: Collagen content in the rat urinary bladder subjected to infravesical outflow obstruction. J Urol 1984; 132: 587-590.

4. Dixon JS, Gilpin CJ, Gilpin SA, Gosling JA, Brading AF, Speakman MJ: Sequential morphological changes in the pig detrusor in responses to chronic partial urethral obstruction. Br J Urol 1989; 64: 385-390.

5. Nielsen KK, Andersen CB, Petersen LK, Oxlund H, Nordling J: Morphological, stereological, and biochemical analysis of the mini-pig urinary bladder after chronic outflow obstruction and after recovery from obstruction. Neurourol Urodyn. 1995; 14: 269-284.

6. Cortivo R, Pagano F, Passerini G, Abatangelo G, Castellani I: Elastin and collagen in the normal and obstructed urinary bladder. Br J Urol 1981; 53: 134137.

7. Kuhn C, Boldt J, King T, Crouch E, Vartio T, Mcdonald $\mathrm{J}$ : An immunohistochemical study of architectural remodeling and connective tissue synthesis in pulmonary fibrosis. Am Rev Respir Dis 1989; 140: 1693-1703.

8. Peten E, Striker L, Carome M, Elliott S, Yang CW, Striker G: The contribution of increased collagen synthesis to human glomerulosclerosis: A quantitative analysis of $\alpha 2 \mathrm{IV}$ collagen mRNA expression by competitive polymerase chain reaction. J Exp Med 1992, 176: 1571-1576.

9. Gilpin S, Gosling J, Barnard R: Morphological and morphometric studies of the human obstructed, 
trabeculated urinary bladder. Br J Urol 1985; 57: 525 529.

10. Abrams P, Blaivas JG, Stanton SL, Andersen JT: The standardization of terminology of lower urinary tract function. Scand J Urol Nephrol 1988; 14 (Suppl): 5-19.

11. Pinheiro AC, Costa WS, Cardoso LE, Sampaio FJ: Organization and relative content of smooth muscle cells, collagen and elastic fibers in the corpus cavernosum of rat penis. J Urol 2000; 164: 1802-1806.

12. Weibel ER, Kistler GS, Scherle WF: Practical stereological methods for morphometric cytology. J Cell Biol 1966; 30: 23-38.

13. Junqueira LCU, Montes GS, Sanchez EM: The influence of tissue section thickness on the study of collagen by the Picrosirius-polarization method. Histochemistry 1982; 74: 153-156.

14. Mayo ME, Lloyd-Davies RW, Shuttleworth KED, Tighe JR: The damaged human detrusor: functional and electron microscopic changes in disease. Br J Urol 1973; 45: 116-125.

15. Gosling JA: Modification of bladder structure in response to outflow obstruction and ageing. Eur Urol 1997; 32 (suppl 1): 9-14.

16. Kim JC, Yoon JY, Seo SI, Hwang TK, Park YH: Effects of partial outlet obstruction and its relief on types I and III collagen and detrusor contractility in the rat. Neurourol Urodyn 2000; 19: 29-42.

Waldemar S. Costa

Urogenital Research Unit - UERJ

Av. 28 de Setembro, 87 - fundos - FCM - terreo

20551-030, Rio de Janeiro, RJ, Brazil

Fax: + $55212587-6121$

E-mail:wscosta@gmail.com

\section{EDITORIAL COMMENT}

Bladder outlet obstruction produces histological changes in the detrusor, including an increased collagen concentration in adult and fetal bladders (1-4). The authors of this paper have
17. Gosling JA, Dixon JS: Detrusor morphology in relation to bladder outflow obstruction and instability. In Hinman FJr., editor. Benigh prostatic hypertrophy. New York, Springer. 1983, pp 666-671.

18. Freedman AL, Qureshi F, Shapiro E, Lepor H, Jacques SM, Evans MI, Smith CA, Gonzalez R, Johnson MP: Smooth muscle development in the obstructed fetal bladder. Urology 1997; 49: 104-107.

19. Lepor H, Sunaryadi I, Hartanto V, Shapiro E: Quantitative morphometry of the adult human bladder. J Urol 1992; 148: 414-417.

20. Holm NR, Horn T, Hald T: Bladder wall morphology in ageing and obstruction. Scand J Urol Nephrol 1995; 29: 45-49.

21. Deveaud CM, Macarak EJ, Kucich U, Ewalt DH, Abrams WR, Howard PS: Molecular analysis of collagens in bladder fibrosis. J Urol 1998; 160: 15181527.

22. Inui E, Ochiai A, Nasa Y, Ukimura O, Kojima M: Comparative morphometric study of bladder detrusor between patients with benigh prostatic hyperplasia and controls. J Urol 1999; 161: 827-830.

23. Collado A, Batista E, Gelabert-Mas A, Corominas JM, Arano P, Villavicencio H: Detrusor quantitative morphometry in obstructed males and controls. J Urol. 2006; 176: 2722-8.

Accepted after revision: September 14, 2006

corroborated that the components of connective tissue are increased in the detrusor muscle of patients with infravesical obstruction. While this is not the first study regarding the association between bladder wall 
collagen deposition and bladder outlet obstruction, it is one of the first report to demonstrate a clear demarcation in collagen content and elastic systems fiber of the obstructed and unobstructed adult human male bladder. This study may enable us to better understand the human bladder reaction to bladder outlet obstruction. Studies of this type should be encouraged.

However, much more work needs to be done to establish a link between lower urinary tract symptoms and this histological finding. First, although they stated that the greenish color present in the sections observed under polarization microscopy suggests the presence of a significant amount of type III collagen, a separate quantification of type I and type III collagen was not performed and no analysis of collagen content by symptom type was reported. Second, no mention was made of compliance measurements. This is of potential since patients with a low compliant bladder expect to have a significant increase in connective tissue compared to those without a low compliant bladder (5). It would have certainly been useful to determine what level of bladder wall collagen is associated with detectable

\section{EDITORIAL COMMENT}

In our urological practice, it is commonly found that patients with profound bladder outlet obstruction (BOO) may have a low bladder compliance. The low compliant bladder contributes to a high intravesical pressure and endangers upper urinary tract function. Moreover, in the patients with low bladder compliance, the detrusor contractility is usually inadequate to completely empty the bladder during micturition. The increased connective tissue density changes in bladder compliance. This work would add to the growing body of evidence linking specific changes in detrusor structure or function with lower urinary tract symptoms. Finally, the increase in the connective tissue may be age-dependent (4). Thus, it would be better to include elderly patients without bladder outlet obstruction in the study.

\section{REFERENCES}

1. Susset JG, Servot-Viguier D, Many F, Madernas P, Black R: Collagen in 155 human bladders. Invest Urol. 1978; 16: 204-6.

2. Uvelius B, Mattiasson A: Collagen content in the rat urinary bladder subjected to infravesical outflow obstruction. J Urol. 1984; 132: 587-90.

3. Kim KM, Kogan BA, Massad CA, Huang YC: Collagen and elastin in the obstructed fetal bladder. J Urol. 1991; 146: 528-31.

4. Lepor H, Sunaryadi I, Hartanto V, Shapiro E: Quantitative morphometry of the adult human bladder. J Urol. 1992; 148: 414-7.

5. Ohnishi N, Kishima Y, Hashimoto K, Kiwamoto H, Esa A, Sugiyama T, et al.: Morphometric study of low compliant bladder. Hinyokika Kiyo 1994; 40: 657-61.

Dr. Jae-Seung Paick Department of Urology Seoul National University Hospital Seoul, Korea E-mail: jspaick@snu.ac.kr

in the bladder wall may not only constrict the distensibility of the urinary bladder but also result in low bladder contractility.

In this article, the authors found the collagen and elastic system fibers increase in the bladder wall in the patients with urodynamically proven BOO compared to the bladders in the non obstructed cadavers. Previous studies have shown in patients with BOO and ageing, the collagen content 
increases. The results of this study are compatible with the previous works using rat models and human bladders and further demonstrate that the connective tissue components are increased in the bladder wall of $\mathrm{BOO}$ in a group of patients with a mean age of 60 years.

Bladder "fibrosis" results in trabeculation, reduced capacity, increased intravesical pressure and suppressed detrusor contractility. This pathological change of the bladder wall is commonly found in varying diseases including neurogenic bladder, BOO, ageing and chronic cystitis. The true mechanisms for the increased connective tissue density in the bladder wall have not been completely elucidated yet. Recent studies have shown that BOO may induce increase in nerve growth factor (NGF) production in the suburothelium, which might result in a cascade of inflammatory reactions, lowering sensory threshold and remodeling of the micturition pathways (1). The increased NGF levels in bladder afferent pathways could contribute to the emergence of bladder overactivity as well as somal hypertrophy and hyperexcitability of bladder afferent neurons (2). It is interesting to find that in patients with transient $\mathrm{BOO}$, the bladder compliance will turn to normal after relief of obstruction, however, in patients with chronic BOO, the changes of the bladder wall will not. Neural plasticity induced increase of connective tissue density in the bladder wall after BOO and the factors responsible for their reversibility deserve further investigations.

\section{REFERENCES}

1. Steer WB, Tuttle JB: Mechanisms of Disease: the role of nerve growth factor in the pathophysiology of bladder disorders. Nat Clin Pract Urol. 2006; 3: 10110.

2. Yoshimura N, Bennett NE, Hayashi Y, Ogawa T, Nishizawa O, Chancellor MB, et al: Bladder overactivity and hyperexcitability of bladder afferent neurons after intrathecal delivery of nerve growth factor in rats. J Neurosci. 2006; 26: 10847-55.

Dr. Hann-Chorng Kuo

Dept. Urology, Buddhist Tzu Chi General Hospital Buddhist Tzu Chi University

Hualien, Taiwan

E-mail:hck@tzuchi.com.tw 